\title{
More on Recombination and Selection in the Modifier Theory of Sex-Ratio Distortion ${ }^{1}$
}

\author{
Marcus W. Feldman and Sarah P. Otto \\ Department of Biological Sciences, Stanford University, \\ Stanford, California 94305
}

Received July 29,1988

\author{
THIS PAPER IS DEDICATED TO THE MEMORY OF SURESH JAYAKAR
}

\begin{abstract}
G. Maffi and S. D. Jayakar suggested a model for the two-locus control of sex determination in the mosquito Aedes aegypti (1981, Theor. Pop. Biol. 19, 19-36). This model was extended to multiple alleles and analyzed in mathematical detail by S. Lessard (1987, Theor. Pop. Biol. 31, 339-358). The model supposes that males are "Mm" and females "mm" but the transmission from males is controlled by a second gene with alleles $A_{i}$. We show that in addition to the equilibrium in which $m A_{i}$ in females, $M A_{i}$ from males and $m A_{i}$ from males all have the same frequencies, a second class of polymorphic equilibria exists and can be stable. The former class was shown by Lessard to be stable for intermediate and/or loose linkage. The new class of equilibria may be stable for tight linkage under the conditions that preclude stability of the former. We also develop the theory of linkage modification from the neighborhood of the new equilibrium. Successful modifiers of recombination may either reduce or increase the recombination fraction with the outcome depending on the linkage of the modifier to the major genes. 1989 Academic Press, Inc.
\end{abstract}

\section{INTRODUCTION}

In several insect species there is sex-ratio distortion as a consequence of sex-linked meiotic drive. Perhaps the most widely known case is that of the $S R$ chromosomal polymorphism in Drosophila pseudoobscura (see Curtsinger and Feldman, 1980). Another well-known example occurs in the mosquito Ades agypti, in which genetic distortion of the sex ratio was originally found by Hickey and Craig (1966).

Maffi and Jayakar (1981) proposed and analyzed a two-locus two-allele model for sex determination inspired by the biology of $A$. agypti. In this species there appears to be a sex-determining locus, identified by Giemsa banding, with alleles $M$ and $m$ such that $M m$ individuals are males and $\mathrm{mm}$

${ }^{1}$ Research supported by NIH grants GM 28016 and GM 10452, and by the Morrison Institute for Population and Resource Studies. 
females. Linked to the $M / m$ band is another band which perturbs the sexratio of males to females in the progeny of $\mathrm{Mm}$ individuals away from oneto-one. Maffi and Jayakar's study entailed two alleles, $A_{1}$ and $A_{2}$, at this distortion producing locus. Lessard (1987) generalized this model to include an arbitrary number of alleles at the sex-ratio modifying locus. Both treatments represent a simplification of the known biology of the situation in $A$. cogypti, and some of the genetically complicating phenomena have been described by Wood (1976) and Newton et al. $(1976,1978)$.

In the studies by both Maffi and Jayakar, and Lessard, recursions were developed for the evolution of the chromosome frequencies in terms of the transmission probabilities of $M$ - and $m$-bearing gametes from males, and the recombination fraction between the $M / m$ and $\left\{A_{i}\right\}$ loci. The conclusions of Maffi and Jayakar were based mainly on numerical iteration of the recursions, while Lessard made an extensive mathematical analysis of certain features of the multi- $A$-allele version of Maffi and Jayakar's model. (Lessard went on to develop a multiple-allele version of Curtsinger and Feldman's (1980) theory of sex-ratio distortion that includes fertility and viability selection. We shall not be concerned with this aspect of the subject in the present note.)

Both of these studies concluded that when the linkage between the two loci is sufficiently tight, no stable isolated equilibrium exists. Lessard's Result IV states that, for small recombination, every equilibrium of the sexratio distortion recursion system is unstable, and that only limit cycles or chaotic behaviors occur. The numerical examples illustrated by Maffi and Jayakar appear to confirm this claim. In this article, we demonstrate that this claim is incorrect and that for tight linkage, and indeed for all $r \in\left[0, \frac{1}{2}\right]$, it is possible that a class of equilibria, identified numerically by Maffi and Jayakar and analytically, in a special case, by Karlin and Lessard (1986), be stable.

We also discuss some results concerning genetic modification of the recombination between $M / m$ and $\left\{A_{i}\right\}$ controlled by a third locus, and examine the parallels between these results and the findings of Thomson and Feldman (1974) in a related model of segregation distortion. The Reduction principle (Feldman and Liberman, 1986; Liberman and Feldman, 1986; Altenberg and Feldman, 1987) is violated in a manner which, we conjecture, is characteristic of models with segregation distortion. We find that although tight linkage between the major and modifier genes generally ensures selection for reduced recombination, when the modifier is further from the major genes, interesting new phenomena appear. For example, we observe that viability-analogous Hardy-Weinberg (VAHW) equilibria may be stable for intermediate values of the recomhination between major and modifier genes, a result quite different from that of Liberman and Feldman (1986). 


\section{The Model and Previous Results}

To describe the main conclusions of these previous studies, it is sufficient to consider two alleles $A_{1}$ and $A_{2}$ at the distortion-modifying locus. Females are of genotypes $m A_{i} / m A_{j}$ and males of genotype $M A_{i} / m A_{j}$ with $i, j=1,2$. The probabilities that $M$ and $m$ are transmitted to the offspring of $M A_{i} / m A_{j}$ are $s_{i j}$ and $1-s_{i j}$, respectively, where we assume $0<s_{i j}=s_{j i}<1$ for $i, j=1,2$. The recombination fraction between $M / m$ and $A_{1} / A_{2}$ is $r$ with $0 \leqslant r \leqslant \frac{1}{2}$. Suppose that $x_{1}$ and $x_{2}=1-x_{1}$ are the frequencies of $m A_{1}$ and $m A_{2}$, respectively, among all gametes transmitted by females, $y_{1}$ and $y_{2}=1-y_{1}$ are the frequencies of $M A_{1}$ and $M A_{2}$, respectively, among all $M$ gametes transmitted by males and $z_{1}$ and $z_{2}=1-z_{1}$ are the frequencies of $m A_{1}$ and $m A_{2}$, respectively, among all $m$ gametes transmitted by males.

The recursions that express the chromosome frequencies $x_{i}^{\prime}, y_{i}^{\prime}, z_{i}^{\prime}$ in the next generation are (see Lessard, 1987)

$$
\begin{aligned}
& x_{1}^{\prime}=\left(x_{1}+z_{1}\right) / 2, \\
& y_{1}^{\prime}=\left[(1-r) y_{1} x_{1 .}+r x_{1} y_{1 .}\right] / \bar{s}, \\
& z_{1}^{\prime}=\left[r y_{1}\left(1-x_{1 .}\right)+(1-r) x_{1}\left(1-y_{1 .}\right)\right] /(1-\bar{s})
\end{aligned}
$$

where

$$
x_{i .}=\sum_{j} s_{i j} x_{j}, \quad y_{i .}=\sum_{j} s_{i j} y_{j}
$$

and

$$
\bar{s}=\sum_{i} y_{i} x_{i .}=\sum_{i} x_{i} y_{i .}=\sum_{i} \sum_{j} s_{i j} x_{i} y_{j} .
$$

Maffi and Jayakar (1981) showed that if $s_{12}>s_{11}, s_{22}$ or $s_{12}<s_{11}, s_{22}$, an equilibrium of the form

$$
\hat{x}_{1}=\hat{y}_{1}=\hat{z}_{1}=\left(s_{12}-s_{22}\right) /\left(2 s_{12}-s_{11}-s_{22}\right)
$$

exists. Lessard (1987) produced the multiple-allele analogue of (2) which has the form of the polymorphism characteristic of viability selection on a single locus. Uyenoyama and Feldman (1981) have called such equilibria "viability analogous." In the context of sex-ratio theory, such equilibria in which the allele frequencies (or chromosome frequencies) are the same in each sex have been called symmetric (Eshel and Feldman, 1982). We shall use the latter terminology here for the equilibrium (2). This equilibrium was shown by Maffi and Jayakar to be unstable at $r=0$. Lessard provided explicit bounds to an interval of the recombination fraction in which (2) is 
stable. This interval excludes $r=0$, and its extremities are expressed as functions of the eigenvalues of a certain matrix. In the case of tight linkage, numerical iteration of (1) by Maffi and Jayakar revealed cyclic behavior of the chromosome frequencies, with no convergence to isolated equilibria. The analysis by Lessard, in this case, suggests that this cyclic behavior for small $r$ results from a Hopf bifurcation that occurs as $r$ decreases across the lower stability bound.

For loose linkage, that is, for $r$ greater than the upper bound for stability of (2), Maffi and Jayakar's numerical analysis produced two additional polymorphic equilibria. The existence of these also emerges from the anaiysis by Lessard, although in neither study are they explicitly obtained. One of the cases examined numerically by Maffi and Jayakar was treated in considerable analytical detail by Lessard and Karlin (1982) and Karlin and Lcssard (1986, pp. 190-192). This casc has $s_{11}=s_{12}$, and Karlin and Lessard exhibit a nonsymmetric polymorphic equilibrium which exists when fixations in $A_{1} A_{1}$ and $A_{2} A_{2}$ are locally unstable. Of course, in this case the symmetric point (2) cannot exist. For our findings here, the interesting fact about Karlin and Lessard's equilibrium is that it exists when $s_{12}>\frac{1}{2}$ and $r<\left(s_{12}-s_{22}\right) /\left(2 s_{12}-1\right)$.

A final point that will be useful in our discussion concerns conditions for stability of allelic or chromosomal fixation states. At $r=0$, Maffi and Jayakar note that $\hat{x}_{1}=\hat{y}_{1}=\hat{z}_{1}=0$, corresponding to fixation in $A_{2}$, is stable if $s_{22}>s_{12}$ while $\hat{x}_{1}=\hat{y}_{1}=\hat{z}_{1}=1$, fixation in $A_{1}$, is stable if $s_{11}>s_{12}$. Two other boundary equilibria were discovered by Maffi and Jayakar to exist for $r=0$. These were the points $\hat{x}_{1}=\hat{z}_{1}=1, \hat{y}_{1}=0$ and $\hat{x}_{1}=\hat{z}_{1}=0$, $\hat{y}_{1}=1$, both of which were classed as "trivial," and were not subjected to further investigation. The present paper focuses on these two equilibria, their stability, and what happens to them as $r$ increases. Attention is restricted to the case of two alleles at the $A$ locus because the basic phenomena emerge in this case. There is no doubt that there will be analogous behavior with multiple alleles at the $A$ locus.

\section{Equilibrium ANALysis}

From (1a) it is obvious that at every equilibrium of (1), $x_{i}=z_{i}$. Make this substitution in (1b) and (1c), then expand (1c) to obtain

$$
y_{1}=\frac{\left(s_{22}-s_{12}\right) x_{1}\left(1-x_{1}\right)-r\left(1-s_{12}\right) x_{1}}{\left(s_{11}+s_{22}-2 s_{12}\right) x_{1}\left(1-x_{1}\right)-r\left(1-s_{12}\right)} .
$$

Substitute (3) into (1b) and the result is a fifth-degree polynomial in $x_{1}$, two of whose roots are $\hat{x}_{1}=0, \hat{x}_{1}=1$. A third factor produces the 
symmetric equilibrium (2). What remains is the quadratic equation $Q\left(r, x_{1}\right)=0$, with

$$
\begin{aligned}
Q\left(r, x_{1}\right)= & x_{1}^{2}\left[\left(s_{12}-s_{11}\right)\left(s_{12}-s_{22}\right)+r s_{12}\left(s_{11}+s_{22}-2 s_{12}\right)\right] \\
& +x_{1}\left[\left(s_{12}-s_{11}\right)\left(s_{22}-s_{12}\right)-r s_{12}\left(s_{11}+s_{22}-2 s_{12}\right)\right. \\
& \left.-r\left(1-s_{12}\right)\left(s_{22}-s_{11}\right)\right]+r\left(1-s_{12}\right)\left[s_{22}-s_{12}-r\left(1-2 s_{12}\right)\right] .
\end{aligned}
$$

We shall call valid roots of (3) and (4) "asymmetric" equilibria.

Obviously $Q\left(0, x_{1}\right)=0$ is the quadratic equation

$$
\left(s_{12}-s_{11}\right)\left(s_{12}-s_{22}\right)\left(x_{1}^{2}-x_{1}\right)=0 .
$$

We investigate the shape of $Q\left(r, x_{1}\right)$ for small $r$ by considering $\partial Q(r, 0) / \partial r$ at $r=0$ and $\partial Q(r, 1) / \partial r$ at $r=0$. In fact

$$
\begin{aligned}
& \left.\frac{\partial Q(r, 0)}{\partial r}\right|_{r=0}>0 \quad \text { if } \quad s_{22}>s_{12}, \\
& <0 \text { if } s_{22}<s_{12}, \\
& \left.\frac{\partial Q(r, 1)}{\partial r}\right|_{r=0}>0 \quad \text { if } \quad s_{11}>s_{12} \text {, } \\
& <0 \text { if } s_{11}<s_{12} .
\end{aligned}
$$

It is then obvious that $Q\left(r, x_{1}\right)=0$ has the following properties for $r$ small:

(a) For $s_{11}>s_{12}, s_{22}>s_{12}$ there are two roots, one close to $x_{1}=0$, one close to $x_{1}=1$.

(b) For $s_{11}<s_{12}, s_{22}<s_{12}$ there are no valid roots.

(c) For $s_{11}>s_{1}>s_{3}$ there is a single root near $x_{1}=0$.

(d) For $s_{22}>s_{12}>s_{11}$ there is a single root near $x_{1}=1$.

It is clear from the comparison of (4) and (5) that as $r$ increases from zero, these asymmetric equilibria emanate from the boundary equilibria $\hat{x}_{1}=\hat{z}_{1}=0, \hat{y}_{1}=1$ and $\hat{x}_{1}=\hat{z}_{1}=1, \hat{y}_{1}=0$ which were two of the "trivial" points noted by Maffi and Jayakar. At the point $\hat{x}_{1}=\hat{z}_{1}=0, \hat{y}_{1}=1$ two gametes are present, $M A_{1}$ and $m A_{2}$. At $\hat{x}_{1}=\hat{z}_{1}=1, \hat{y}_{1}=0$ the two gametes in the population are $M A_{2}$ and $m A_{1}$. Clearly these two points are analogous to the so-called "high complementarity" equilibria (Franklin and Lewontin, 1970) of classical two-locus theory at which linkage disequilibrium is maximized as a function of the recombination fraction. The symmetric equilibrium corresponds to the "central" equilibrium at which gametic association is zero. 
The argument so far has concerned the validity of the roots of (4). But for these values of $\hat{x}_{1}$ to be valid, they must also produce values of $\hat{y}_{1}$ from (3) which are valid. We need not consider the case $s_{12}>s_{11}, s_{22}$ where for small $r$ no valid roots of (4) exist. The other three cases, (a), (c), and (d) of the previous paragraph, remain to be examined. First notice that from $Q\left(r, x_{1}(r)\right)=0$ with $x_{1}(r)=r x_{1}^{\prime}(0)+O\left(r^{2}\right)$ we have

$$
\frac{\partial Q(0,0)}{\partial r}+x_{1}^{\prime}(0) \frac{\partial Q(0,0)}{\partial x_{1}}=0 \text {. }
$$

This then entails that

$$
x_{1}^{\prime}(0)=\left(1-s_{12}\right) /\left(s_{11}-s_{12}\right),
$$

so that for small $r$, the smaller root of (4) is

$$
\hat{x}_{1}^{(s)}=\frac{r\left(1-s_{12}\right)}{s_{11}-s_{12}}+O\left(r^{2}\right)
$$

A similar argument near $x_{1}=1$ produces the larger root as

$$
\hat{x}_{1}^{(l)}=1-\frac{r\left(1-s_{12}\right)}{s_{22}-s_{12}}+O\left(r^{2}\right) .
$$

At $x_{1}=r\left(1-s_{12}\right) /\left(s_{11}-s_{12}\right)$ substitution into (4) reveals that if $s_{22}>s_{12}$, $s_{11}>s_{12}$ then $Q\left[r, r\left(1-s_{12}\right) /\left(s_{11} \cdots s_{12}\right)\right]<0$ for small $r$, while if $s_{11}>s_{12}>s_{22}$ then $Q\left[r, r\left(1-s_{12}\right) /\left(s_{11}-s_{12}\right)\right]>0$. In both cases the shape of the quadratic (4) entails that the smaller root $\hat{x}_{1}^{(s)}$ is actually smaller than $r\left(1-s_{12}\right) /\left(s_{11}-s_{12}\right)$.

Now consider the denominator of $y_{1}$ in (3), which can be expressed, using (8) as

$$
\frac{\left(s_{22}-s_{12}\right) r\left(1-s_{12}\right)}{s_{11}-s_{12}}+O\left(r^{2}\right)
$$

If $s_{22}>s_{12}, s_{11}>s_{12},(10)$ is positive and $\hat{y}_{1}<1$ if $\left(s_{11}-s_{12}\right) \hat{x}_{1}>r\left(1-s_{12}\right)$, which contradicts the previous paragraph. If $s_{11}>s_{12}>s_{22}$ then (10) is negative and $\hat{y}_{1}<1$ if $\left(s_{11}-s_{12}\right) \hat{x}_{1}<r\left(1-s_{12}\right)$, which is compatible with the previous paragraph. We conclude that when $s_{11}>s_{12}>s_{22}$ and $r$ is small, the asymmetric root corresponding at $r=0$ to $\hat{x}_{1}=0$ moves into the interior of the frequency simplex. When $s_{11}, s_{22}>s_{12}$ this root moves out of the simplex and is biologically invalid. A similar argument applies to the larger root (9), which is valid for small $r$ if $s_{22}>s_{12}>s_{11}$ and inadmissible for small $r$ if $s_{11}, s_{22}>s_{12}$. 
Remark. Lessard and Karlin (1982) and Karlin and Lessard (1986, pp. 190-192) consider a particular case originally treated by Maffi and Jayakar, namely, $s_{11}=s_{12}$. In this case, under either of the conditions $s_{22}<s_{12}, 2 s_{12}>1$ or $s_{22}>s_{12}, 1>2 s_{12}$ there is a single valid root of (4) and (5) provided $r\left(1-2 s_{12}\right) /\left(s_{22}-s_{12}\right)<1$. Under these conditions, both fixations $\hat{x}_{1}=\hat{y}_{1}=\hat{z}_{1}=0$ and $\hat{x}_{1}=\hat{y}_{1}=\hat{z}_{1}=1$ are locally unstable. (Note that Karlin and Lessard (p. 192) refer only to the case $s_{22}<s_{12}, 2 s_{12}>1$, but, for example, when $s_{22}=0.6, s_{12}=s_{11}=0.4$ and $r=0.2$, the point $\hat{x}_{1}=\hat{z}_{1}=0.64792, \hat{y}_{1}=0.43195$ is a valid equilibrium.)

\section{Stability Considerations}

\subsection{The Symmetric Point and Fixations Do Not Overlap in Stability}

From Karlin and Lessard (1986) the condition for the stability of the fixation state $\hat{x}_{1}=\hat{y}_{1}=\hat{z}_{1}=0$ is

$$
r>\frac{s_{22}-s_{12}}{1-2 s_{12}}
$$

provided $1>2 s_{12}, s_{22}>s_{12}$ or $1<2 s_{12}, s_{22}<s_{12}$. For an admissible range of recombination values, this entails that $s_{22}<\frac{1}{2}$ in the case $s_{12}<\frac{1}{2}$, and $s_{22}>\frac{1}{2}$ in the case $s_{12}>\frac{1}{2}$. Let us concentrate on the case

$$
\frac{1}{2}>s_{22}>s_{12}, \quad r>\frac{s_{22}-s_{12}}{1-2 s_{12}},
$$

in which case the fixation point is stable. In the Appendix, we show that the symmetric equilibrium

$$
\hat{x}_{1}=\hat{y}_{1}=\hat{z}_{1}=\frac{s_{22}-s_{12}}{s_{11}+s_{22}-2 s_{12}}
$$

cannot be stable in the range of recombination values for which this fixation point is stable. An alternative proof for this can be deduced from Lessard's (1987) Result I.

\subsection{Stability of the Asymmetric Equilibria}

Consider the point $\hat{x}_{1}=\hat{z}_{1}=1, \hat{y}_{1}=0$. This is locally stable if $s_{11}<s_{12}<s_{22}$, while $\hat{x}_{1}=\hat{z}_{1}=0, \hat{y}_{1}=1$ is locally stable if $s_{11}>s_{12}>s_{22}$. If these inequalities hold, then by continuity for $r$ sufficiently small, the corresponding equilibria (which have moved slightly away from the boundary into the interior of the frequency simplex by virtue of recombination) 
are stable. In each case there is a single stable asymmetric equilibrium for sufficiently tight linkage.

For larger values of the recombination fraction, the local stability analysis of the asymmetric equilibria, even in the two-allele case, is algebraically very formidable. We shall report the results of extensive numerical iteration of system (1) according to the different arrangements of the sex-ratio determining parameters. These parameters were each chosen from a uniform distribution on $(0,1)$ and the ordering of $s_{11}, s_{12}, s_{22}$ assigned at random. For each ordered set, 20 initial frequencies were inserted into the iteration of (1), which was carried out for an array of recombination values including $r=0$ and $r=0.5$. The following is a summary of the equilibrium patterns observed as $r$ increased from 0 to 0.5 .

Case 1. $s_{11}>s_{12}>s_{22}$. Here, at $r=0$, the only stable fixed point was $\hat{x}_{1}=\hat{z}_{1}=0, \hat{y}_{1}=1$. As $r$ increased, $\hat{x}_{1}=\hat{z}_{1}$ increased and $\hat{y}_{1}$ decreased continuously as the equilibrium moved into the interior of the simplex, and this asymmetric polymorphic equilibrium was globally stable for all $r \in[0,0.5]$. With $s_{22}>s_{12}>s_{11}$, the results here were as just described, except that the fixed point at $r=0$ was given by $\hat{x}_{1}=\hat{z}_{1}=1, \hat{y}_{1}=0$. Again as $r$ increased, $\hat{x}_{1}=\hat{z}_{1}$ decreased and $\hat{y}_{1}$ increased, and there was a unique globally stable polymorphic equilibrium for $r \in[0,0.5]$.

Case 2. $s_{11}>s_{12}>s_{22} ; s_{22}>s_{12}>s_{11}$. Here, in both cases, there was a value $r_{0}$, such that for $r<r_{0}$, the unique asymmetric equilibrium was globally stable, while for $r>r_{0}$, one of the fixation states became stable and remained stable for all higher $r$.

Case 3. $s_{11}>s_{12}, s_{22}>s_{12}$ or $s_{12}>s_{11}, s_{12}>s_{22}$. These classes are considered together because insofar as the behavior of the asymmetric equilibria for moderate and loose linkage is concerned, they appeared to be similar. In every numerical case treated, the findings of Maffi and Jayakar and Lessard for tight linkage were confirmed; as $r$ increased from zero, there was cycling which stopped for a value $r_{0}$ of the recombination fraction $\left(r_{0}\right.$ depended on $\left.\left\{s_{i j}\right\}\right)$ beyond which the symmetric equilibrium became stable. It remained stable for an interval of recombination values that depended on $\left\{s_{i j}\right\}$. We observed cases in which this interval extended to $r=0.5$, with no other stable equilibria revealed. Of more interest here are the cases in which (as predicted by Lessard) the symmetric equilibria gave way at some value $r^{*}$ to two asymmetric equilibria, namely, the roots of (4) with (3). Three patterns of behavior were observed as $r$ increased depending on the values of $\left\{s_{i j}\right\}$ :

(i) The asymmetric equilibria could remain stable for all $r \in\left[r^{*}, \frac{1}{2}\right]$.

(ii) At some value $\tilde{r}_{1}<\frac{1}{2}$, one of the asymmetric equilibria disappeared, giving way to one of the two corners $\hat{x}_{1}=\hat{y}_{1}=\hat{z}_{1}=0$ or 
$\hat{x}_{1}=\hat{y}_{1}=\hat{z}_{1}=1$, and at a value $\tilde{r}_{2}$ with $\tilde{r}_{1}<\tilde{r}_{2}<\frac{1}{2}$ the other asymmetric equilibrium gave way to the other corner. Thus, in the recombination interval $\left(\tilde{r}_{1}, \tilde{r}_{2}\right)$, one asymmetric polymorphism and one fixation state were simultaneously stable, and in $\left(\tilde{r}_{2}, \frac{1}{2}\right)$ the two fixations were stable.

(iii) A number of cases were observed in which $\tilde{r}_{2}$ extended to $\frac{1}{2}$. In this case, the two fixation states were never simultaneously stable. Of course, the conditions predicting when one or both of the fixations become stable are given by (11), and its analogue with $s_{11}$ substituted for $s_{22}$.

\section{Genetic Modification of Recombination with Sex-Ratio Distortion}

Consider a third gene with alleles $f_{1}$ and $f_{2}$ such that the genotype $f_{1} f_{1}$ produces a recombination fraction $r_{11}$ between the $M / m$ and $A_{1} / A_{2}$ loci, with $r_{12}$ and $r_{22}$ the recombination fractions corresponding to $f_{1} f_{2}$ and $f_{2} f_{2}$. The evolution of recombination, posed initially in this way by Nei (1967), has been studied by Feldman (1972), Feldman et al. (1980), and Liberman and Feldman (1986) in terms of the initial increase properties of allele $f_{2}$ near fixation of $f_{1}$. The population is originally fixed on $f_{1}$, and chromosomes $f_{1} M A_{1}, f_{1} M A_{2}, f_{1} m A_{1}, f_{1} m A_{2}$ evolve under the interacting forces of recombination $\left(r_{11}\right)$ between $M$ and $A$ and whatever selection acts on the $M$ and $A$ genes. In the neighborhood of the equilibrium attained with $f_{1}$ fixed, $f_{2}$ is introduced in low frequency and the modification question boils down to determination of conditions for the initial increase of $f_{2}$. In the present case, the selection is due to the distortion matrix $\left\|s_{i j}\right\|$. (See Karlin and Lessard (1986) for a discussion of the relationship between sex determination and two-sex viability models.)

It is obvious that for recombination between $M / m$ and $A_{1} / A_{2}$ and initial increase at the $f_{1} / f_{2}$ locus to be related, there must be linkage disequilibrium between the two former genes. In other words, prior to the introduction of $f_{2}$, the population with $f_{1}$ fixed must evolve toward one of the asymmetric equilibria. In the neighborhood of this equilibrium, $f_{2}$ is introduced and we seek conditions that are sufficient for it to increase in frequency. In deterministic models with Mendelian segregation, constant viability selection, and random mating, the Reduction principle, which states that $f_{2}$ increases when rare if $r_{12}<r_{11}$, seems to be a general result independent of the viability regime and of the degree of linkage between $f_{1} / f_{2}$ and the other two loci (Liberman and Feldman, 1986). The one previous model for the evolution of recombination in the presence of segregation distortion produced departures from the Reduction principle. To place the present work in context, we summarize these earlier results.

Thomson and Feldman (1974) examined the recombination-modification question for a two-gene model of segregation distortion originally proposed 
by Prout et al. (1973). In that model, a locus with alleles $M$ and $m$ controlled the segregation in heterozygotes at another gene $D / d$ which was also subject to viability selection. Suppose that the recombination fraction between the recombination controlling gene $f_{1} / f_{2}$ and $D / d$ is $s$, and $f_{1} f_{1}$, $f_{1} f_{2}, f_{2} f_{2}$ produce recombination fractions $r_{11}, r_{12}, r_{22}$, respectively, between $D / d$ and $M / m$, and that there is no interference. Then Thomson and Feldman showed that, when the ordering of the genes was $F-D-M$, the initial increase of $f_{2}$ when rare depended on $s$. Thus, $f_{2}$ increased when rare provided

$$
\text { (i) } 0 \leqslant s<r_{11} \quad \text { and } \quad r_{12}<r_{11}
$$

or

$$
\text { (ii) } s>r_{11} \quad \text { and } \quad r_{12}>r_{11} \text {. }
$$

Conditions (12) and their analogs for increase of $f_{1}$ in the neighborhood of $f_{2}$-fixation produce the following picture for the evolution of the $f_{1} / f_{2}$ locus.

Case (i). $r_{11}<r_{12}<r_{22}$. Starting near fixation of $f_{1}, f_{2}$ is eliminated if $s<r_{11}$ and $f_{2}$ increases if $s>r_{11}$. Starting near fixation of $f_{2}, f_{1}$ increases if $s<r_{22}$ and is lost if $s>r_{22}$. Thus for $r_{11}<s<r_{22}$ we expect a polymorphism at the recombination-controlling locus.

Case (ii). $\quad r_{11}<r_{22}<r_{12}$. Here when $s>r_{22}$ both $f_{1}$ and $f_{2}$ increase when rare and polymorphism for $f_{1}$ and $f_{2}$ is expected.

Case (iii). $r_{12}<r_{11}<r_{22}$. Here if $s<r_{11}$ both $f_{1}$ and $f_{2}$ increase when rare and polymorphism is expected.

The other possible orderings of $r_{11}, r_{12}, r_{22}$ are qualitatively contained in these three cases. These simple bounds on $s$, in terms of $r_{11}$ and $r_{22}$, are probably a result of the simple selection scheme considered by Thomson and Feldman, which was the one Prout et al. (1973) analyzed most extensively. More complicated criteria for initial increase might well emerge from the model that incorporates the complete parameter set of Prout et al. (1973) as treated later by Thomson and Feldman (1976). Nevertheless, the simple parameter set suffices to produce violations of the Reduction principle and the existence of polymorphisms at $f_{1} / f_{2}$, even when there is directional ordering of the recombination rates.

We now present the results of our study of the evolution of recombination controlling alleles in the model of sex-ratio distortion. Analytical results have been difficult to obtain, but we present a few, and then proceed to outline the results of a numerical study. A qualitative pattern emerges which is in some ways similar to that described above for the segregation distortion model, but which also has a number of interesting complications. 


\subsection{Analytical Results concerning Initial Increase of Recombination Modifiers}

With two alleles at each of the three loci, the local stability of fixation in $f_{1}$, where the recombination fraction is $r_{11}$, to invasion by $f_{2}$ is determined by the eigenvalues of a $6 \times 6$ matrix which we refer to as $\mathbf{L}$. The recombination fraction for $f_{1} f_{2}$ is $r_{12}$ and the first analytical result is that, when $r_{11}=r_{12}$ and interference is sufficiently low, the largest eigenvalue of $\mathbf{L}$ is unity. This is a consequence of the fact that the determinant $|\mathbf{I}-\mathbf{L}|$ vanishes and each of the five principal minors of $\mathbf{I}-\mathbf{L}$ is positive (Gantmacher, 1959, pp. 68-72). In the presence of arbitrary interference, the determinant $|\mathbf{I}-\mathbf{L}|$ vanishes, but we have not been able to demonstrate the positivity of the principal minors. The algebraic details of these facts are omitted.

When $s=0$ (and there is no interference), $\mathbf{L}$ splits into a $2 \times 2$ and a $4 \times 4$ matrix. After some algebra, it is possible to show that the leading eigenvalue of the $2 \times 2$ matrix is greater than unity if $r_{12}<r_{11}$, less than unity if $r_{12}>r_{11}$ and, according to the previous paragraph, equal to unity if $r_{11}=r_{12}$. Exactly the same is also true of the $4 \times 4$ block of $L$. Hence for $s=0$, and indeed for $s$ sufficiently small, an allele that reduces recombination will invade. The dynamics subsequent to invasion are of considerable interest and the next result describes a class of polymorphisms for recombination-controlling alleles.

Polymorphism at loci which control recombination as well as mutation and migration has been a focus of our recent work on modifiers (Feldman and Liberman, 1986). In light of our discoveries about polymorphism in these models, which do not involve departures from Mendelian segregation, the following result which holds for the present case of sex-ratio distortion, as well as for Prout et al.'s general model of segregation distortion, is of some interest.

\subsection{Existence of the Viability-Analogous Hardy-Weinberg Equilibrium}

Suppose that $r_{12}<r_{11}, r_{22}$ or $r_{12}>r_{11}, r_{22}$. Then in the standard onelocus selection model with viabilities represented by $r_{11}, r_{12}, r_{22}$, a polymorphic equilibrium exists with the frequencies of $f_{1}$ and $f_{2}$ given by $\hat{p}_{1}$ and $\hat{p}_{2}$ with

$$
\begin{aligned}
& \hat{p}_{1}=\left(r_{22}-r_{12}\right) /\left(r_{11}+r_{22}-2 r_{12}\right), \\
& \hat{p}_{2}=\left(r_{11}-r_{12}\right) /\left(r_{11}+r_{22}-2 r_{12}\right),
\end{aligned}
$$

and equilibrium mean viability given by

$$
r^{*}=\hat{p}_{1}^{2} r_{11}+2 \hat{p}_{1} \hat{p}_{2} r_{12}+\hat{p}_{2}^{2} r_{22}
$$


Suppose also that an asymmetric equilibrium of the recursion system (1) exists (there may be two of these) for the recombination rate $r^{*}$ and is given by $x_{1}^{*}, y_{1}^{*}, z_{1}^{*}=x_{1}^{*}$. Then there is an equilibrium of the full threelocus system with frequencies

$$
\begin{aligned}
& \left\{x_{1}^{*} \hat{p}_{1}, x_{2}^{*} \hat{p}_{1}, y_{1}^{*} \hat{p}_{1}, y_{2}^{*} \hat{p}_{1}, z_{1}^{*} \hat{p}_{1}=x_{1}^{*} \hat{p}_{1}, z_{2}^{*} \hat{p}_{1}=x_{2}^{*} \hat{p}_{1},\right. \\
& \left.x_{1}^{*} \hat{p}_{2}, x_{2}^{*} \hat{p}_{2}, y_{1}^{*} \hat{p}_{2}, y_{2}^{*} \hat{p}_{2}, z_{1}^{*} \hat{p}_{2}=x_{1}^{*} \hat{p}_{2}, z_{2}^{*} \hat{p}_{2}=x_{2}^{*} \hat{p}_{2}\right\} .
\end{aligned}
$$

We have termed this a viability-analogous Hardy-Weinberg equilibrium to emphasize that the recombination rates behave as viabilities and that there is linkage equilibrium between $f_{1} / f_{2}$ and the two-locus system $\left\{\boldsymbol{M} / m, A_{1} / A_{2}\right\}$.

Remark 1. In our previous studies of VAHW equilibria for recombination modifiers with Mendelian segregation, we have proved that these equilibria are internally stable or unstable according to whether $s$ is sufficiently large or small, respectively (Feldman and Krakauer, 1976; Liberman and Feldman, 1986).

Remark 2. If, as in Case (i) of the segregation-distortion study by Thomson and Feldman, there is a polymorphism with $r_{11}<r_{12}<r_{22}$ it cannot be VAHW. Polymorphisms that do not have the VAHW structure have been detected before in models with Mendelian segregation, but have proven to be particularly difficult to analyze (Feldman and Balkau, 1973).

\subsection{Numerical Results on Evolution of Recombination Modifiers}

In order to study the dynamics of recombination modification for large values of $s$, general distortion $\left\|s_{i j}\right\|$ matrices were chosen uniformly on $(0,1)$ and tested for existence of stable asymmetric equilibria for some range of recombination rates in the two-locus system. Values of $r_{11}, r_{12}, r_{22}$ were then chosen so that either $r_{11}$, or $r_{22}$, or both were within this range. Numerical iteration of the full three-locus system was then commenced from initial frequencies close to the asymmetric equilibrium corresponding either to fixation of $f_{1}\left(r_{11}\right)$ or of $f_{2}\left(r_{22}\right)$. In a number of cases iteration was begun from initial frequencies near the VAHW if it existed. In all cases the fate of $f_{1}$ and $f_{2}$ was tracked for an array of $s$ values in $\left[0, \frac{1}{2}\right]$. In all cases we found it difficult to detect patterns of evolutionary behavior at the $f_{1} / f_{2}$ locus that reflected the ordering of the distortion patterns $s_{11}, s_{12}, s_{22}$. We were able, however, to distinguish patterns that reflected the ordering of $r_{11}, r_{12}, r_{22}$. We develop these in terms of the three orderings $r_{11}<r_{12}<r_{22}$, $r_{11}<r_{22}<r_{12}, r_{12}<r_{11}<r_{22}$. 
Case 1. Directional, $r_{11}<r_{12}<r_{22}$. As demonstrated analytically, for $s$ small $f_{2}$ is eliminated when initially rare. In fact, for small $s$ the population is globally attracted to fixation in $f_{1}$. In some cases this outcome was observed for $s \leqslant \frac{1}{2}$, but in others there were values $\hat{s}$ and $\tilde{s}$ such that for $\hat{s}<s<\tilde{s}$ the population was globally attracted to a polymorphic equilibrium. This polymorphism, which of course is not VAHW, is of considerable interest. It is characterized by varying with $s$ and by the identities

$$
\begin{array}{lll}
f_{1} m & \text { from females }=f_{1} m & \text { from males, } \\
m A_{1} & \text { from females }=m A_{1} & \text { from males. }
\end{array}
$$

In view of the dependence of this nonVAHW equilibrium on $s$, it is not surprising that we have been unable to obtain an analytic expression for it. In most cases where $\tilde{s}<\frac{1}{2}$, with $\tilde{s}<s<\frac{1}{2}$ the population fixed globally on $f_{2}$, although in one case both asymmetric equilibria corresponding to fixation in $f_{1}$ and $f_{2}$ were stable for $s$ close to 0.5 . In some cases we failed to observe the interval $(\hat{s}, \tilde{s})$. This may simply mean that our parameter grid was not fine enough, or that in fact there is a sharp discontinuity in $s$ above and below which high and low recombination, respectively, are favored.

Case 2. $r_{11}<r_{22}<r_{12}$. Recall that in the case of Mendelian segregation, the VAHW equilibrium, which may exist in this case, is not stable when the heterozygote has the highest recombination rate. For $s$ small, neither $f_{1}$ or $f_{2}$ can increase when rare. There are two values of $s$ which we denote ${ }_{1} s$ and ${ }_{2} s$ such that for $s<{ }_{1} s, f_{2}$ is lost when rare and for $s<{ }_{2} s, f_{1}$ is lost when rare. For $s<s^{*}=\min \left({ }_{1} s,{ }_{2} s\right)$ both fixation in $f_{1}$ and $f_{2}$ are stable. It is possible that $s^{*} \geqslant \frac{1}{2}$ and that this is the whole picture. More often, however, $s_{v}=\max \left({ }_{1} s,{ }_{2} s\right)<\frac{1}{2}$ and for $\frac{1}{2} \geqslant s>s_{v}$, the population converges globally to the VAHW equilibrium. In these examples, for $s \in\left({ }_{1} s,{ }_{2} s\right), f_{2}$ increases from near fixation in $f_{1}$ and the population converges to a nonVAHW equilibrium, while from near fixation in $f_{2}, f_{1}$ is lost. Thus for $s \in\left({ }_{1} s,{ }_{2} s\right)$ the nonVAHW polymorphic equilibrium and fixation in $f_{2}$ are simultaneously stable.

Case 3. $r_{12}<r_{11}<r_{22}$. In all cases, at $s=0$ the population converged to one of two "high complementarity" equilibria. These have either $f_{1} m A_{1}$, $f_{1} m A_{2}, f_{2} M A_{1}, f_{2} M A_{2}$ present with the first two equally frequent in males and females, and the frequencies of $m A_{1}$ and $M A_{1}$ equal to those at an asymmetric equilibrium with recombination rate $r_{12}$, or the analogous arrangement with $f_{1} M A_{1}, f_{1} M A_{2}, f_{2} m A_{1}, f_{2} m A_{2}$. For $0<s \leqslant{ }_{3} s$ the VAHW equilibrium was globally stable and in some cases ${ }_{3} s$ extended to 0.5 . When $\frac{1}{2}>{ }_{3} s$ two values ${ }_{1} s \geqslant{ }_{3} s$ and ${ }_{2} s \geqslant{ }_{3} s$ were observed such that for 
$s>_{1} s$, fixation in $f_{1}$ was stable and for $s>{ }_{2} s$, fixation in $f_{2}$ was stable. For $s>\max \left({ }_{1} s,{ }_{2} s\right)$ both fixations were stable, and in some cases we found ${ }_{3} s={ }_{1} s={ }_{2} s$. (This may be an artifact of an insufficiently fine search for nonVAHW equilibria.) When ${ }_{3} s<\min \left({ }_{1} s,{ }_{2} s\right)$, then for $s \in\left({ }_{3} s, \min \left({ }_{1} s,{ }_{2} s\right)\right)$ we observe two distinct nonVAHW stable equilibria. For $s \in\left({ }_{1} s,{ }_{2} s\right)$ fixation in $f_{1}$ was simultaneously stable with a single nonVAHW equilibrium.

\section{Discussion}

The symmetric and asymmetric equilibria of the two-locus sex-ratio distortion model have stability properties analogous to those of the equilibria with linkage equilibrium and linkage disequilibrium in the segregation distortion model of Prout et al. (1973). The symmetric equilibrium is stable in an interval of recombination values always bounded away from zero, and often bounded away from one half. By contrast, with Mendelian segregation, if $D=0$ is stable for some $r_{0}$, it is stable for $r>r_{0}$. In this case when a polymorphism with $D \neq 0$ is stable, it generally occurs for tight linkage. In the sex-ratio distortion case we observe that asymmetric equilibria can be stable for $r$ large. In addition, in cases where, for example, $s_{11}>s_{12}>s_{22}$, our new finding is that asymmetric equilibria may be stable near $r=0$. These asymmetric equilibria appear to bifurcate from high complementarity equilibria at $r=0$, such as the one in which $m A_{1}$ and $M A_{2}$ are the only chromosomes represented. Overall, the general finding with Mendelian segregation, that equilibrium values of $D$ decrease with increasing recombination, appears to be violated in the presence of segregation distortion.

A similar departure from Mendelian expectation characterizes our results on recombination modification. Although the findings are not as simple as those of Thomson and Feldman (1974), there are some important similarities. First, the Reduction principle is valid for sufficiently tight linkage between the modifier and the major genes. For loose linkage, however, the principle is violated in interesting ways.

Viability-analogous Hardy-Weinberg equilibria have been shown to exist in the presence of segregation distortion. However, we have seen that their stability properties are dramatically different from those we demonstrated with Mendelian segregation. Thus, VAHW equilibria may be stable in a range of $s$ values bounded away from $\frac{1}{2}$. Further, depending on the ordering of $r_{11}, r_{12}, r_{22}$, another class of equilibria polymorphic for the recombination modifier may be stable for $s$ on either side of the range of stability of the VAHW equilibrium.

Our analysis has been carried out with two alleles at each locus. There is every reason to believe that the anomalous equilibrium properties 
catalogued here will also arise when there are multiple $A$-alleles and multiple alleles at the recombination controlling locus. Altenberg and Feldman (1987) suggested that these anomalies are connected with the "nonlinear" mathematical structure of the transmission law of chromosomes from generation to generation. With segregation distortion models, it would be useful to develop a more precise understanding of the mathematical underpinnings of the breakdown in reduction properties.

\section{APPENDIX 1}

The local stability matrix for the symmetric equilibrium is given as Table I. We begin by showing that under the conditions which produce stability of the fixation state, at $r=\frac{1}{2}$ this fixation and the symmetric point cannot both be stable. Lessard has several results concerning what happens at $r=\frac{1}{2}$. In the two-allele case these are considerably simpler.

The reason for this is that the local stability matrix simplifies in this case so that the 2, 1 element equals the 2,2 element and the 3,1 element equals the 3,2 element at $r=\frac{1}{2}$. As a result, the value of the characteristic cubic at $\lambda=1$ reduces to

$$
\frac{1}{2}\left[1-s_{12} / 2 \bar{s}-\left(1-s_{12}\right) /(2(1-\bar{s}))\right]
$$

where $\bar{s}$ is the equilibrium fraction of males, namely,

$$
\bar{s}=\left(s_{11} s_{22}-s_{12}^{2}\right) /\left(s_{11}+s_{22}-2 s_{12}\right) .
$$

Stability of the central point requires that

$$
2 \bar{s}(1-\bar{s})-s_{12}(1-\bar{s})-\bar{s}\left(1-s_{12}\right)<0 .
$$

We now show that under the conditions stated above, this is impossible. It should be noted that the matrix in Table I may not be positive, so that condition (A2) is not, in general, sufficient for stability of the central point.

TABLE I

Local Stability Matrix ${ }^{a}$

\begin{tabular}{ccc}
\hline $1 / 2$ & 0 & $1 / 2$ \\
$\left(\hat{\hat{s}}^{-1}\right)\left[\hat{y}_{1}\left(1-\hat{y}_{1}\right)\left(s_{11}+s_{22}-2 s_{12}\right)+r s_{12}\right]$ & $(\hat{\bar{s}}-1)\left[\left(1-\hat{y}_{1}\right) x_{1}+\hat{y}_{1} x_{2}-r s_{12}\right]$ & 0 \\
$(1-\hat{\bar{s}})^{-1}\left[1-y_{1 .}\left(1-\hat{x}_{1}\right)-y_{2} \hat{x}_{1}-r\left(1-s_{12}\right)\right]$ & $(1-\hat{\bar{s}})^{-1}\left[-\hat{x}_{1}\left(1-\hat{x}_{1}\right)\left(s_{11}+s_{22}-2 s_{12}\right)+r\left(1-s_{12}\right)\right]$ & 0 \\
\hline
\end{tabular}

$a \hat{\bar{s}}$ is the value of $\sum_{i} \sum_{j} s_{i j} \hat{x}_{i} \hat{y}_{j}$ at the equilibrium $\left\{\hat{x}_{i}\right\},\left\{\hat{y}_{i}\right\}$. 
Algebraic expansion of the left side of (A2) produces

$$
\begin{aligned}
& \left(\bar{s}-s_{12}\right)(1-2 \bar{s}) \\
& =\frac{-\left(s_{11}-s_{12}\right)\left(s_{22}-s_{12}\right)\left[2 s_{11} s_{22}-2 s_{12}^{2}-s_{11}-s_{22}+2 s_{12}\right]}{\left(s_{11}+s_{22}-2 s_{12}\right)^{2}},
\end{aligned}
$$

which is negative if the last bracket in the numerator is positive. Clearly for this we require

$$
s_{11}\left(2 s_{22}-1\right)+2 s_{12}\left(1-s_{12}\right)-s_{22}>0
$$

or, since $s_{22}<\frac{1}{2}$,

$$
s_{11}<\frac{2 s_{12}\left(1-s_{12}\right)-s_{22}}{1-2 s_{22}} .
$$

Now for the existence of the symmetric point, it is necessary that $s_{11}>s_{12}$ (since by assumption $s_{22}>s_{12}$ ). Thus, (A4) must be compatible with $s_{11}>s_{12}$. We show that this is impossible, and in fact

$$
s_{12}>\frac{2 s_{12}\left(1-s_{12}\right)-s_{22}}{1-2 s_{22}} .
$$

To see that $(A 5)$ is true, note that it reduces to

$$
s_{12}\left(1-2 s_{22}\right)>2 s_{12}\left(1-s_{12}\right)-s_{22}
$$

or

$$
s_{12}\left(1-2 s_{12}\right)<s_{22}\left(1-2 s_{12}\right),
$$

which is true since $s_{12}<s_{22}$. We conclude that at $r=\frac{1}{2}$, overlap in stability is impossible.

I now show that, in fact, the symmetric equilibrium

$$
\hat{x}_{1}=\hat{y}_{1}=\hat{z}_{1}=\frac{s_{22}-s_{12}}{s_{11}+s_{22}-2 s_{12}}
$$

is unstable at

$$
r=\frac{s_{22}-s_{12}}{1-2 s_{12}} \quad \text { and } \quad r=\frac{s_{11}-s_{12}}{1-2 s_{12}}
$$

Return to the matrix in Table I. It is easy to see that the value of the characteristic polynomial is linear in the recombination fraction. If this 
value is positive at $r=\left(s_{22}-s_{12}\right) /\left(1-2 s_{12}\right)$ and $r=\left(s_{11}-s_{12}\right) /\left(1-2 s_{12}\right)$ then, since it is positive at $r=\frac{1}{2}$, it is positive in the interval

$$
\left\lceil\min \left(\frac{s_{22}-s_{12}}{1-2 s_{12}}, \frac{s_{11}-s_{12}}{1-2 s_{12}}\right), \frac{1}{2}\right],
$$

in which case the symmetric point is unstable in this range.

The value of the characteristic polynomial at $\lambda=$ unity is

$$
\frac{1}{2}\left\{\alpha \beta+r\left[\beta \frac{s_{12}}{\bar{s}}+\frac{\alpha\left(1-s_{12}\right)}{1-\bar{s}}\right]\right\},
$$

where

$$
\begin{aligned}
& \alpha=\frac{\hat{x}_{1}\left(1-\hat{x}_{1}\right)\left(s_{11}+s_{22}-2 s_{12}\right)}{\bar{s}} \\
& \beta=\frac{-\hat{x}_{1}\left(1-\hat{x}_{1}\right)\left(s_{11}+s_{22}-2 s_{12}\right)}{1-\bar{s}} .
\end{aligned}
$$

We expand (A6) as

$$
\begin{aligned}
\frac{1}{2}\left\{\frac{-\hat{x}_{1}^{2}\left(1-\hat{x}_{1}\right)^{2}\left(s_{11}+s_{22}-2 s_{12}\right)^{2}}{\bar{s}(1-\bar{s})}\right. & \left.+r\left[\frac{-s_{12} \hat{x}_{1}\left(1-\hat{x}_{1}\right)+\left(1-s_{12}\right) \hat{x}_{1}\left(1-\hat{x}_{1}\right)}{\bar{s}(1-\bar{s})}\right]\left(s_{11}+s_{22}-2 s_{12}\right)\right\} \\
= & \frac{\hat{x}_{1}\left(1-\hat{x}_{1}\right)\left(s_{11}+s_{22}-2 s_{12}\right)}{2 \bar{s}(1-\bar{s})} \\
& \times\left\{-\hat{x}_{1}\left(1-\dot{x}_{1}\right)\left(s_{11}+s_{22}-2 s_{12}\right)+r\left(1-2 s_{12}\right)\right\} \\
= & \frac{\hat{x}_{1}\left(1-\hat{x}_{1}\right)\left(s_{11}+s_{22}-2 s_{12}\right)}{2 \bar{s}(1-\bar{s})} \\
& \times\left\{\frac{-\left(s_{11}-s_{12}\right)\left(s_{22}-s_{12}\right)}{s_{11}+s_{22}-2 s_{12}}+\left(s_{22}-s_{12}\right)\right\} \\
= & \frac{\hat{x}_{1}\left(1-\hat{x}_{1}\right)\left(s_{11}+s_{22}-2 s_{12}\right)}{2 \bar{s}(1-\bar{s})} \\
& \times\left\{\frac{\left(s_{22}-s_{12}\right)^{2}}{\left(s_{11}+s_{22}-2 s_{12}\right)}\right\} \\
> & 0
\end{aligned}
$$


in the case $r=\left(s_{22}-s_{12}\right) /\left(1-2 s_{12}\right)$. When $r=\left(s_{11}-s_{12}\right) /\left(1-2 s_{12}\right)$ interchange $s_{22}$ and $s_{11}$ in (A7), with the same positive result. This concludes the proof that the fixation states and the symmetric point cannot overlap in stability.

\section{ACKNOWLEDGMENTS}

The authors are grateful to Professors Sabin Lessard and Uri Liberman for their careful criticism of an earlier draft.

\section{REFERENCES}

Altenderg, L., and Feldman, M. W. 1987. Selection, generalized transmission and the evolution of modifier genes. I. The reduction principle, Genetics 117, 559-572.

Curtsinger, J. W., and Feldman, M. W. 1980. Experimental and theoretical analysis of the "sex-ratio" polymorphism in Drosophila pseudoobscura, Genetics 94, 445-466.

Eshel, I., and Feldman, M. W. 1982. On evolutionary genetic stability of the sex ratio, Theor. Pop. Biol. 21, 430-439.

Feldman, M. W. 1972. Selection for linkage modification. I. Random mating populations, Theor. Pop. Biol. 3, 324-346.

Feldman, M. W., and B. BalKaU, 1973. Selection for linkage modification. II. A recombination balance for neutral modifiers, Genetics 74, 713-726.

Feldman, M. W., Christiansen, F. B., and Brooks, L. 1980. Evolution of recombination in a constant environment, Proc. Nat. Acad. Sci. USA 77, 4838-4841.

Feldman, M. W., and Krakauer, J. 1976. Genetic modification and modifier polymorphisms, in "Population Genetics and Ecology" (S. Karlin and E. Nevo, Eds.), Academic Press, New York.

Feldman, M. W., and Liberman, U. 1986. An evolutionary reduction principle for genetic modifiers, Proc. Natl. Acad. Sci. USA 83, 4824-4827.

Franklin, I., AND Lewontin, R. C. 1970. Is the gene the unit of selection? Genetics 65, 701-734.

Gantmacher, F. R. 1959. "The Theory of Matrices." Vol. II, Chelsea, New York.

Hickey, W. A., and Craig, G. B. JR. 1966. Genetic distortion of sex ratio in a mosquito, Edes agypti, Genetics 53, 1177-1196.

Karlin, S., and Lessard, S. 1986. "Theoretical Studies on Sex Ratio Evolution," Princeton Univ. Press, Princeton, NJ.

LESSARD, S. 1987. The role of recombination and selection in the modifier theory of sex-ratio distortion, Theor. Pop. Biol. 31, 339-358.

LESSARD, S., AND KARLIN, S. 1982. A criterion for stability-instability at fixation states involving an eigenvalue one with applications in population genetics, Theor. Pop. Biol. 22, $108-126$.

Liberman, U., AND FeldMan, M. W. 1986. A general reduction principle for genetic modifiers and recombination, Theor. Pop. Biol. 30, 341-371.

MAFFi, G., and JaYaKaR, S. D. 1981. A two-locus model for polymorphism for sex-linked meiotic drive modifiers with possible applications to Ades agypti, Theor. Pop. Biol. 19, $19-36$.

NEI, M. 1967. Modification of linkage intensity by natural selection, Genetics 57, 625-641. 
Newton, M. E., Wood, R. J., And Southern, D. I. 1976. A cytogenetic analysis of meiotic drive in the mosquito, Ades agypti, (L.), Genetica 46, 297-318.

Newton, M. E., Woov, R. J., And Southere, D. I. 1978. Cytological mapping of the $M$ and $D$ loci in the mosquito, Edes agypti (L.), Genetica 48, 137-143.

Prout, T., BundgaArd, J., ANd Bryant, S. 1973. Population genetics of modifiers of meiotic drive. I. The solution of a special case and some general implications, Theor. Pop. Biol. 4, 446-465.

Thomson, G. J., and Feldman, M. W. 1974. Population genetics of modifiers of meiotic drive. II. Linkage modification in the segregation distortion system, Theor. Pop. Biol. 5 , $155-162$.

Thomson, G. J., and Feldman, M. W. 1976. Population genetics of modifiers of meiotic drive. III. Equilibrium analysis of a general model for the genetic control of segregation distortion, Theor. Pop. Biol. 10, 10-25.

Uyenoyama, M. K., and Feldman, M. W. 1981. On relatedness and adaptive topography in kin selection, Theor. Pop. Biol. 19, 87-123.

WoOD, R. J. 1976. Between family variation in sex ratio in the Trinidad (T-30) strain of AEdes agypti $\left(\mathrm{L}\right.$.) indicating differences in sensitivity to the meiotic drive gene $M^{\mathrm{D}}$, Genetica $\mathbf{4 6}$, 345-361. 